Geistige Eigentumsrechte, was steht für die Entwicklungsländer auf dem spiel?

\title{
Arzneimittelzugang und TRIPS-Abkommen
}

\section{Pascale Brudon}

\section{(2) OpenEdition \\ 1 Journals}

Electronic version

URL: http://journals.openedition.org/sjep/715

DOI: 10.4000/sjep.715

ISSN: 1663-9677

Publisher

Institut de hautes études internationales et du développement

\section{Printed version}

Date of publication: 1 avril 1998

Number of pages: $87-96$

ISSN: 1660-5926

\section{Electronic reference}

Pascale Brudon, «Arzneimittelzugang und TRIPS-Abkommen », Schweizerisches Jahrbuch für Entwicklungspolitik [Online], 17 | 1998, Online erschienen am: 04 August 2012, abgerufen am 08 September 2020. URL : http://journals.openedition.org/sjep/715; DOI : https://doi.org/10.4000/sjep. 715 
T

ROTZ einer regelmässigen und ständigen Verbesserung des Gesundheitswesens in der Welt, auch in den meisten Entwicklungsländern, sterben jedes Jahr 17 Millionen Menschen, darunter eine Mehrzahl von Kindern unter fünf Jahren, an übertragbaren Krankheiten, die oft durch eine aktive Verhütungspolitik vermieden oder durch eine angemessene Therapie behandelt und geheilt werden könnten (Infektions- und Parasitenkrankheiten). Wegen einer raschen Alterung der Bevölkerung in den entwickelten Ländern, aber auch in den Entwicklungsländern, leiden immer mehr Menschen an nicht übertragbaren chronischen Krankheiten, die lange Behandlungen erfordern. Neue Krankheiten sind in den letzten Jahren aufgetreten - wie AIDS - und andere, die seit langem bekannt waren und manchmal sogar unter Kontrolle standen wenn nicht ausgerottet waren, breiten sich in vielen Ländern wieder aus (Tuberkulose, Malaria, usw.). Schliesslich wirft die zunehmende Resistenz zahlreicher Infektionsträger (Bakterien, Parasiten), welche Verursacher der in den Entwicklungsländern vorherrschenden Krankheiten (Malaria, akute Infektionen der Atemwege, Tuberkulose usw.) und der Nosokomialinfektionen in den entwickelten Ländern sind, heute schwerwiegende Probleme auf.

Wenn in einem solchen epidemiologischen Umfeld die Verhütung mehr denn je das Hauptinstrument zur wirksamen Krankheitsbekämpfung ist, so spielen die Arzneimittel weiterhin eine wesentliche Rolle, um die Ausbreitung der überwiegenden Krankheiten zu kontrollieren bzw. zu reduzieren und dadurch die Lebenserwartung (unbeeinträchtigt durch Unfähigkeit) und die Lebensqualität zu verbessern. Jedoch sind im Arzneimittelbereich, wie in vielen anderen Bereichen, die Ungleichheiten zwischen den entwickelten Ländern und der übrigen Welt beträchtlich. Der Arzneimittelkonsum, der sich 1995 auf 284 Milliarden Dollar (Herstellerpreis) belief, ist in den entwickelten Ländern konzentriert, da im gleichen Jahr 80\% der in der Welt hergestellten Arzneimittel von 25\% der Weltbevölkerung, nämlich derjenigen in Nordamerika, Europa und Japan konsumiert wurde. ${ }^{1}$ Hingegen haben in den Entwicklungsländern über eine Milliarde Menschen (wahrscheinlich zwei) keinen regelmässigen Zugang zu den unentbehrlichsten Arzneimitteln. ${ }^{2}$ Seit zehn Jahren wurden die - zahlreichen neuen Medikamente zum grössten Teil von einer kleinen Anzahl transnationaler Pharmaunternehmen entdeckt, hergestellt und vermarktet, welche hauptsächlich in den Industrieländern ansässig sind. ${ }^{3}$

Obwohl eine solche Darstellung etwas schematisch und somit notgedrungen unvollständig ist, erscheint es für das Verständnis der Arzneimittelsituation in der Welt bequem, die Länder in drei grosse Gruppen aufzuteilen: die Industrie-

\footnotetext{
* Wissenschafterin, Aktionsprogramm für unentbehrliche Arzneimittel, Weltgesundheitsorganisation (WHO), Genf.

1. Scrip. Nr. 2172, 15. Oktober 1996, S. 14.

2. Rapport sur la santé dans le monde 1996. WHO, Genf, S. 84.

3. Ballance R, Pogány J., Forstner H. The World's Pharmaceutical Industries: An International Perspective on Innovation, Competition and Policy. Wien, United Nations Industrial Development Organization (UNIDO), 1992.
} 
länder, wo Forschung und Produktion konzentriert sind, und die allein (wertmässig) über vier Fünftel der in der Welt hergestellten Arzneimittel konsumieren; einige Entwicklungsländer, die im allgemeinen eine grosse Bevölkerung haben (China, Indien, Mexiko, Brasilien, Argentinien) und denen es gelungen ist, eine nationale Pharmaindustrie bzw. sogar eine gewisse Forschungskapazität zu entwickeln; und schliesslich die reinen Verbraucherländer, welche einige Fertiggüter herstellen, aber die meisten ihrer Medikamente einführen.

Im Rahmen der Uruguay-Runde und im Anhang zum WTO-Rahmenabkommen ist ein Übereinkommen aufgeführt, das für die Arzneimittel von besonderer Bedeutung ist: das Abkommen über handelsbezogene Aspekte der Rechte an geistigem Eigentum (TRIPS). Seine Ziele liegen darin, den Schutz des geistigen Eigentums, darunter das gewerbliche Eigentum, weltweit zu stärken und zu harmonisieren.

Wie wird die Lage der Arzneimittel in den Entwicklungsländern künftig nach der Umsetzung des TRIPS-Abkommens sein? Werden sich die nationalen Arzneimittelpolitiken und die Strategien, die zum Hauptziel haben, die Verfügbarkeit der Medikamente zu verbessern, und die von den internationalen Organisationen unterstützt werden, weiterhin entwickeln können? Wird das Abkommen zu Strategien führen, die eine bessere Zugänglichkeit zu den alten oder neuen unentbehrlichen Arzneimitteln in den Entwicklungsländern ermöglichen und den Technologietransfer fördern? Oder wird dieses Abkommen im Gegenteil eine zusätzliche Belastung für die bereits defizitären Gesundheitsbudgets der Entwicklungsländer und für die Bevölkerung dieser Länder bilden? Ist das TRIPS-Abkommen schliesslich einer besseren Arzneimittelversorgung in den Entwicklungsländern förderlich? Obwohl die Dinge erst am Anfang stehen, scheint es notwendig, die möglichen Auswirkungen dieses Abkommens auf die Arzneimittelpolitiken der Entwicklungsländer zu untersuchen, um eine Weiterentwicklung der Strategien vorzuschlagen, welche die Verfügbarkeit qualitativ guter unentbehrlicher Arzneimittel zu erschwinglichen Kosten erhöhen sollen.

\section{ARZNEIMITTELPOLITIKEN UND SCHUTZ DES GEISTIGEN EIGENTUMS}

\subsection{INDUSTRIELÄNDER}

Das Patentsystem für Arzneimittel hat sich im Laufe der Zeit weiterentwickelt. Je mehr sich die Industrieländer entwickelten, desto strenger war ihr System. Die meisten entwickelten Länder haben ein ziemlich strenges Patentwesen, das im allgemeinen in den 70er Jahren eingeführt wurde. Das System der Vereinigten Staaten schützt die Interessen des Erfinders am besten: zwanzig Jahre Patentschutz für Verfahren ebenso wie für Produkte, keine Verpflichtungen für die Patentinhaber. Letztere können ihr Patent nutzen oder auch nicht, es unter Lizenz vergeben oder nicht. Der Konsens ist jedoch nicht unumschränkt in diesen Ländern; einige Patientengruppen, zum Beispiel die Bejahrten in den USA, sind gegen eine zu lange Schutzdauer, welche die Vermarktung billigerer Produkte verhindert, jedoch geht der Trend in den Industrieländern auf eine Verstärkung und Ausdehnung der Patentschutzdauer hin. Die Gründe für diese Entwicklung sind wahrscheinlich darin zu suchen, dass Patente wichtige Instrumente sind, die es den forschenden Arzneimittelfirmen ermöglichen, ihre Inve- 
stitionen durch Abwälzung auf die Preise wieder hereinzuholen. ${ }^{4}$ Patente erlauben hohe Preise, die ihrerseits Gewinne einbringen, um weiter Forschung zu betreiben, welche wiederum neue Arzneimittel hervorbringt. Nun ist aber in den entwickelten Ländern die tatsächliche Schutzdauer für Arzneimittel aufgrund der vorgeschriebenen Produktvermarktungsbestimmungen zurückgegangen. Zudem hat der Wettbewerb mit dem Aufkommen von Generikaherstellern auf dem Markt zugenommen. Schliesslich beginnt der Markt der Entwicklungsländer, zumindest einiger von ihnen, interessant zu werden. Deshalb gehören die Wiederherstellung eines effektiven Patentschutzes in den entwickelten Ländern und die Einführung eines wirksameren Patentschutzes in den Entwicklungsländern zu den legitimen Strategien der transnationalen Arzneimittelfirmen, um keine Marktanteile zu verlieren und weiterhin immer aufwendigere Forschungstätigkeiten $\mathrm{zu}$ betreiben. ${ }^{5}$

\subsection{ENTWICKLUNGSLÄNDER MIT NATIONALER PHARMAINDUSTRIE}

Gleich nach Ende des Zweiten Weltkriegs haben grosse Länder wie Argentinien, Brasilien, Ägypten, Indien, Mexiko und die Republik Korea Politiken zum Anreiz der lokalen Arzneimittelherstellung eingeführt, um den Medikamentenkonsum auf ihrem Binnenmarkt zu steigern. Die meisten von ihnen konnten ihr Angebot an neuen Medikamenten zu erschwinglichen Preisen durch einen geringen Schutz des geistigen Eigentums erhöhen. Jedoch konnte keines dieser Länder neue Medikamente entdecken. Argentinien und Brasilien hatten keine Patente für pharmazeutische Produkte. Die Firmen konnten die von anderen Firmen erfundenen Arzneimittel kopieren und auf dem nationalen Markt verkaufen, ohnen gegen nationales Gesetz zu verstossen. Eine von vielen Entwicklungsländern gewählte mittlere Position war, nur Produktpatente oder nur Verfahrenspatente zu haben. Ein Erfinder konnte das Verfahren, mit dem er ein Produkt herstellte, nicht aber das Medikament als solches patentieren; eine andere Firma hatte dann die Möglichkeit, das gleiche Medikament herzustellen, wenn sie fähig war, eine andere Produktionsmethode zu entwickeln. Die Länder operierten auch mit der Patentdauer, welche kürzer oder länger sein konnte, und mit der Möglichkeit, Zwangslizenzen einzuführen (der Patentinhaber muss jenen, die das Patent nutzen wollen, gegen angemessene Gebühren nichtausschliessliche Lizenzen erteilen). Schliesslich konnte ein Patentinhaber mittels der Verpflichtung, das Patent zu nutzen, gezwungen werden, das neue Medikament in dem Land, das diese Bedingung auferlegte, herzustellen, andernfalls verlor er sein Patent in diesem Land.

So schaffte das 1970 erlassene indische Gesetz «Indian Patents Act» die Patente für Produkte in gesundheitsrelevanten Bereichen ab und beschränkte die Verfahrensschutzdauer für Arzneimittel auf fünf bis sieben Jahre. Ferner liess das Gesetz die Verwendung von Zwangslizenzen für Artikel zu, die für das öffentliche Gesundheitswesen von Interesse waren. Diese Politik zeitigte ihre Früchte. Ab 1979 stellten einheimische Firmen Arzneimittel her, für die im Ausland Patente erteilt worden waren. 1990 waren mindestens ein Dutzend

4. Nogues J. Social costs and benefits of introducing patent protection for pharmaceutical drugs in developing countries. The Developing Economies, März 1993, Band 31 (1), S. 24-53.

5. Nogues J. Patents and pharmaceutical drugs: understanding the pressures on developing countries. Journal of World Trade 1990, Band 24(6), S. 81-104. 
indischer Firmen imstande, relativ neue Medikamente wie Ciprofloxacin (Antibiotikum) oder Ranitidin (Mittel zur Behandlung von Geschwüren) aufgrund von Produktionsverfahren herzustellen, die sich von den vom Erfinder des Produkts verwendeten Verfahren unterschieden. Die Preise für diese Medikamente waren viel niedriger als diejenigen in der übrigen Welt. Beispielsweise war in Indien der Preis für Ranitidin 16,6 mal niedriger als der Preis, zu dem das Arzneimittel von Glaxo (dem Erfinder des Produkts) in Grossbritannien verkauft wurde. $^{6}$

Desgleichen stellte die Shin Poong Pharmaceutical Company in der Republik Korea 1983 Praziquantel her, ein von zwei deutschen Pharmaunternehmen, Bayer und E. Merck, entdecktes Arzneimittel, das 1973 in Deutschland patentiert und Anfang der 80er Jahre auf dem internationalen Markt in grossem Umfang vertrieben wurde. Praziquantel stellt die wichtigste Entdeckung bei der Behandlung der Schistosomiasis dar, einer in mehr als 70 Ländern endemisch auftretenden Krankheit, an der über 200 Millionen Menschen auf der Welt leiden. Es ist auch das ausgewählte Mittel zur Behandlung anderer Krankheiten, darunter der Chinesische Leberegel (Opisthorchiasis). Anfang der 80er Jahre waren $10 \%$ der koreanischen Bevölkerung vom Chinesischen Leberegel befallen. Das von Bayer auf den Markt gebrachte Medikament war für die Patienten, die hauptsächlich aus den ärmsten Bevölkerungsschichten stammten, finanziell wenig erschwinglich. Daher erhielt die Shin Poong Pharmaceutical Company von der koreanischen Regierung finanzielle Unterstützung, um mit einem öffentlichen Forschungsinstitut zusammen ein neues Herstellungsverfahren zu entwickeln, was für eine verhältnismässig bescheidene Summe (14'000 Dollar) erfolgte. Das neue Verfahren hatte auch den Vorteil, niedrigere Herstellungskosten zu verursachen. Das Produkt wurde 1985 in Korea patentiert und erhielt in zwölf Ländern ein Verfahrenspatent, darunter die Vereinigten Staaten, Japan und Grossbritannien. Diese Entwicklung erlaubte:

口 Einen Preisrückgang für Praziquantel auf dem koreanischen Markt dank einer Wettbewerbs- und Nachfragesteigerung. 1983 betrug der Preis einer von der Shin Poong Pharmaceutical Company unter dem Namen Distocide ${ }^{\circledR}$ vertriebenen Tablette 3,22 US-Dollar, gegenüber 4,83 Dollar für Bayer. 1994 betrug der Preis einer Tablette der Shin Poong Pharmaceutical Company real 2,03 Dollar und der von Bayer 1,84 Dollar.

口 Eine Steigerung der Ausfuhren von Praziquantel als Rohstoff oder Fertigprodukt, insbesondere in China zur Massenbehandlung der Bevölkerung der endemischem Gebiete, aber auch in entwickelten Ländern (Schweiz, Niederlande, usw.).

• Die Herstellung von Praziquantel im Sudan und in Ägypten aufgrund von Abkommen zwischen nationalen Firmen und der Shin Poong Pharmaceutical Company.

口 Nicht unerhebliche gesundheitliche Vorteile für zahlreiche Bewohner der Erde. $^{7}$

6. Bidwai P. One step forward, many steps backward: dismemberment of India's national drug policy. Development Dialogue, 1995, Band 1, S. 193-222.

7. Reich $\mathrm{MR}$, et al. International strategies for tropical disease treatments: experiences with praziquantel. WHO/DAP/97.9. Genf, WHO, 1997. 


\section{3. ÜBRIGE ENTWICKLUNGSLÄNDER}

In diesen Ländern ist die hauptsächliche Politik, die seit Anfang der $80^{\text {er }}$ Jahre unter der Schirmherrschaft der Weltgesundheitsorganisation angewandt wurde, die Politik der unentbehrlichen Arzneimittel. 1977 haben von der WHO einberufene Experten ein Musterverzeichnis mit Medikamenten entwickelt, welche unentbehrliche Arzneimittel genannt wurden und mit denen sich die Mehrheit der Krankheiten behandeln lässt. ${ }^{8}$ Dieses regelmässig auf den neuesten Stand gebrachte Verzeichnis umfasst zwischen 250 und 300 Medikamente mit dem international gebräuchlichen Namen. Die Länder können diese Liste gemäss den bei ihnen auftretenden Krankheiten, ihren Gesundheitsdiensten, der Ausbildung ihres Personals, usw. ändern. Diese Auswahl von Arzneimitteln, zusammen mit Massnahmen wie durch Ausschreibungen und unter dem Freinamen erfolgende Beschaffungen, Interventionen zur Wettbewerbsförderung über die Preise und den Verkauf von Generika, eine bessere Schulung der Verschreiber, und der Öffentlichkeit für den Gebrauch der Arzneimittel usw., erlaubt es, den pharmazeutischen Sektor zu rationalisieren und die Bevölkerung am kostengünstigsten zu behandeln. Über hundert Länder haben jetzt eine Liste unentbehrlicher Arzneimittel. Die meisten davon sind Medikamente, die sich mit der Zeit bewährt haben und daher im allgemeinen nicht mehr durch Patente geschützt sind. Sie können zu sehr wettbewerbsfähigen Preisen auf dem internationalen Markt gekauft werden. Dennoch enthalten die meisten Listen unentbehrlicher Arzneimittel der Entwicklungsländer auch relativ neue Medikamente, die noch unter Patentschutz stehen. Die Zahl dieser neuen unentbehrlichen Arzneimittel kann in den kommenden Jahren nur noch zunehmen wegen Krankheiten wie AIDS (Antiretroviralmittel) und wegen der Resistenz der Mikroorganismen gegenüber den alten Arzneimitteln, insbesondere den Antibiotika. Viele Entwicklungsländer haben vom geringen Schutz des geistigen Eigentums oder vom Fehlen eines globalen Schutzmechanismus profitiert, um diese neuen Arzneimittel aus Ländern einzuführen, die sie mit anderen Produktionsverfahren als denen des Erfinders herstellen und die sie billiger verkaufen.

So haben die Entwicklungsländer, unabhängig von ihrem sozioökonomischen Niveau, versucht, den Arzneimittelzugang für ihre Bevölkerung zu verbessern, indem sie sich unter anderem den geringen Schutz zunutze machten, der Erfindungen in der Welt gewährt wird. Man versteht besser, warum das TRIPSAbkommen Anlass zu einer langen Schlacht zwischen den entwickelten Ländern und den Entwicklungsländern war, wobei erstere ihre Industrie und deren Forschungskapazität schützen wollten und letztere eine nationale Kapazität entwickeln und die Verfügbarkeit von Arzneimitteln verbessern wollten. 1994 haben die meisten Länder ihre Gesetzgebung geändert und den Schutz des geistigen Eigentums für Arzneimittel erhöht. Dies erfolgte grossenteils unter dem Druck der entwickelten Länder. Argentinien, Brasilien, Indien oder Thailand waren Gegenstand von Untersuchungen oder Vergeltungsmassnahmen im Rahmen von Abschnitt 301 des «Trade and Tariffs Act» der amerikanischen Gesetzgebung. ${ }^{9}$

8. La sélection des médicaments essentiels. Rapport d'un comité d'experts de l'OMS. Série de rapports techniques Nr. 615. Genf, WHO, 1997.

9. Correa CM. The Uruguay Round and drugs. WHO Task Force on Health Economics. Genf, WHO, 1997. 


\section{TRIPS-ABKOMMEN UND PHARMAZEUTIKA: WAS WIRD SICH ÄNDERN?}

Das TRIPS-Abkommen enthält Bestimmungen, die für die Arzneimittelsituation in den Entwicklungsländern wichtig sind,${ }^{10}$ insbesondere :

口 Jeder WTO-Mitgliedsstaat muss für jede Erfindung im pharmazeutischen Bereich einen Patentschutz gewähren, ob es sich dabei um Produkte oder Verfahren handelt (Artikel 27.1). Dies bedeutet, dass jede Nachahmung eines patentierten Arzneimittels von nun an untersagt ist, unter Androhung von Handelssanktionen, die vom Streitbeilegungsorgan der WTO verhängt werden. Der gleiche Artikel legt fest, dass die Behandlung von Patentrechten dieselbe sein muss ungeachtet des Ursprungsortes der Erfindung, des Technologiebereichs oder danach, ob die Produkte importiert oder an Ort und Stelle hergestellt werden. Die letztgenannte Bedingung erlaubt es den Ländern nicht mehr, Zwangslizenzen zu erteilen, wenn eine Firma das Patent nicht nutzte. Desgleichen kann ein Land ein ausländisches Unternehmen, das ein Patent besass, nicht mehr zwingen, das Arzneimittel im Land herzustellen, wenn es die Gebühren erhalten wollte. Im Rahmen des neuen Abkommens kann das Unternehmen das Produkt einfach einführen, um die Patentgebühren einzunehmen.

- Die Patente sind für mindestens zwanzig Jahre vom Datum der Einreichung des Patentantrages an geschützt und der Schutz der bestehenden Patente wird ab dem Zeitpunkt verlängert, zu dem das Abkommen vom betreffenden Land angewandt wird (Artikel 33). Die von den Regierungen vor dem 1. Januar 1995 erteilten Zwangslizenzen werden nicht dem System des Abkommens unterworfen (Artikel 70). Die erteilten Rechte schliessen für Produktpatente das Recht ein, «Dritten zu untersagen, ohne Zustimmung des Inhabers folgende Handlungen vorzunehmen: das Herstellen, das Benutzen, das Anbieten, das Verkaufen und das diesen Zwecken dienende Einführen des Erzeugnisses » (Artikel 28). Jedoch wird das durch diesen Artikel eingeräumte ausschliessliche Recht, das patentierte Erzeugnis zu importieren, durch den Artikel 6 des Abkommens eingeschränkt, der die Frage der Erschöpfung der Rechte an geistigem Eigentum vom Anwendungsbereich des Abkommens ausschliesst. Kurz gesagt wird ein Staat dadurch ermächtigt, ein patentiertes Produkt ohne Erlaubnis des Patentinhabers aus jedem anderen WTOMitgliedsstaat einzuführen, wo es legal vertrieben wurde (Parallelimport). Diese Klausel hat zum Ziel, den Wettbewerb zu steigern, da die gleichen Produkte nicht in allen Ländern den gleichen Preis haben.

口 Das TRIPS-Abkommen erlaubt es den Ländern, bei der Ausarbeitung oder Änderung ihrer Gesetze «die Massnahmen zu treffen, die zum Schutz der Volksgesundheit und -ernährung... notwendig sind, sofern diese Massnahmen mit dem Abkommen vereinbar sind» (Artikel 8). Folglich kann ein Staat Ausnahmen vorsehen, wenn er sie für den Schutz der Volksgesundheit für erforderlich hält. Solche Ausnahmen müssen beschränkt und gerechtfertigt sein.

10. WHO/DAP. Mondialisation et accès aux médicaments - les implications de l'accord ADPIC/OMC. Genf, WGO, Publikation in Vorbereitung, 1998. 
- Das Abkommen erlaubt es, das Patent ohne Genehmigung des Patentinhabers zu nutzen (Zwangslizenz), und es zählt die Bedingungen auf, unter denen dies möglich ist. Die Staaten können im Fall des nationalen Notstands, der öffentlichen Nutzung zu nichtgewerblichen Zwecken und zur Abstellung wettbewerbswidriger Praktiken von diesen Bedingungen abweichen.

a Schliesslich soll das TRIPS-Abkommen, das am 1. Januar 1996 in Kraft getreten ist, von den Industrieländern spätestens ein Jahr danach, von den Entwicklungs- und Transitionsländern fünf Jahre danach (am 1. Januar 2000) und von den am wenigsten entwickelten Ländern elf Jahre danach mit Verlängerungsmöglichkeit (2006) angewandt werden. Entwicklungsländer, die vor der Unterzeichnung des Abkommens keine Produktpatente vergaben, haben für die Gewährleistung eines solches Schutzes zehn Jahre Zeit. Während der Übergangsperiode sollen die Länder eine Struktur zur Annahme der Patentanträge für die neuen Arzneimittel errichten (Artikel 70.8). Bis zur Erteilung des Patents werden ausschliessliche Vermarktungsrechte für fünf Jahre nach der Erlangung der Marktzulassung im Land gewährt (Artikel 70.9).

\section{WELCHE FOLGEN WIRD DAS NEUE ABKOMMEN AUF DIE ARZNEIMITTELSITUATION IN DER WELT UND DEN ARTZNEIMITTELZUGANG IN DEN ENTWICKLUNGSLÄNDERN HABEN?}

Da die TRIPS-Abkommen vollständig während der Uruguay-Runde geschaffen wurden, konnten ihre Auswirkungen noch nicht untersucht werden. Dennoch kann man von den Erfahrungen der Vergangenheit ausgehend einige Schlussfolgerungen über ihre möglichen Folgen im Bereich der Medikamente und der Arzneimittelpolitiken ziehen. Diese Folgen werden für die drei oben festgelegten Ländergruppen - Industrieländer, Entwicklungsländer mit nationaler Pharmaindustrie und übrige Entwicklungsländer - nicht die gleichen sein.

\subsection{INDUSTRIELÄNDER}

Die höheren Gewinne, die sich aus einem besseren Schutz des geistigen Eigentums und dem Verschwinden von Produkten ergeben, die mit dem patentierten Arzneimittel im Wettbewerb standen, sollte es den Forschungsfirmen erlauben, mehr Forschung zu finanzieren, neue Medikamente zu entdecken und dadurch ihre Position auf dem internationalen Markt zu stärken. Es ist wahrscheinlich, dass sich die Forschung weiterhin den Gesundheitsproblemen der entwickelten Länder zuwenden wird. Einige dieser neuen Arzneimittel, wie die Antiretroviralmittel zur Behandlung von AIDS, werden sicher sowohl im Süden und als auch im Norden nützlich sein. Jedoch zeigt der Fall des Praziquantels, dass die Entdeckung eines neuen Medikaments nicht unbedingt seine Verfügbarkeit für die Kranken nach sich zieht; dies gilt besonders für die Armen in den Entwicklungsländern. Zum anderen wird sich die Forschung der transnationalen Firmen, in Ermangelung einer zahlungsfähigen Nachfrage in den Ländern des Südens, nicht ohne Anreize den wesentlichen Problemen dieser Länder zuwenden. 


\subsection{ENTWICKLUNGSLÄNDER MIT NATIONALER PHARMAINDUSTRIE}

Drei grosse Themen beherrschen die Debatte über die Vor- und Nachteile des Schutzes des geistigen Eigentums für diese Ländergruppe:

\section{Wird das TRIPS-Abkommen die Forschung fördern?}

Die Forschungs- und Entwicklungskosten für ein neues Molekül wurden von der pharmazeutischen Industrie auf rund 300 Millionen Dollar geschätzt;" wenige Arzneimittelfirmen der Entwicklungsländer haben ein genügendes Verkaufsvolumen, um solche Investitionen vorzunehmen. Obwohl heute eine Reihe bedeutender Entwicklungsländer die wissenschaftlichen Kompetenzen besitzen, um bereits auf dem Markt bestehende Produkte durch ein neues Verfahren herzustellen, sind sich zahlreiche Autoren zum anderen einig, dass der Erfolg im Rahmen der Innovation, wie sie bei den neuen Medikamenten stattfindet, mehr mit dem wirtschaftlichen Entwicklungsstand eines Landes als mit seinem Patentschutzsystem zusammenhängt. Kein Land von mittlerem sozioökonomischem Niveau mit einem Produktpatentschutz hat neue Medikamente erfunden. ${ }^{12,13}$ Die Erfindung von Arzneimitteln scheint eher das Resultat der wirtschaftlichen Entwicklung als ein Faktor dieser Entwicklung zu sein. Im übrigen haben die meisten entwickelten Länder erst in einem ziemlich späten Stadium ihrer Entwicklung ein Patentsystem eingeführt und die Pharmaindustrie hat von den Anstrengungen in der Grundlagenforschung profitiert und profitiert weiterhin davon.

Kurzfristig werden lokale Firmen, die patentgeschützte Arzneimittel herstellen und vermarkten möchten, dem Patentinhaber Gebühren zahlen müssen und werden nicht mehr die Möglichkeit haben, Forschungstätigkeiten zu entwickeln, um neue Herstellungsverfahren zu entdecken: der Fall des Medikaments Praziquantel zeigt, das dank dieses fehlenden Produktschutzes eine grössere Anzahl von Schistosomiasis-Patienten behandelt werden konnten, da die Preise auf dem Weltmarkt erheblich gesunken waren (vgl. Anmerkung 7). Langfristig besteht die Wahrscheinlichkeit, dass einige Unternehmen der grossen Entwicklungsländer fähig sein werden, die Herausforderung anzunehmen und mit der Unterstützung ihrer Regierung Forschungstätigkeiten aufzunehmen, die zur Entdeckung neuer Moleküle führen. ${ }^{14}$

\section{$\square$ Wird das TRIPS-Abkommen den Technologietransfer fördern?}

Bisher zwangen die meisten nationalen Gesetzgebungen ein Unternehmen, das ein Patent besass, das Medikament im Land herzustellen oder Lizenzen zu erteilen, wenn es seine Gebühren einnehmen wollte. Importieren genügte nicht. Mit den TRIPS-Abkommen genügt es, zu importieren, um die Patentgebühren einzunehmen. Nach Meinung einiger Autoren werden die Arzneimittelfirmen es vorziehen, ihre Produktion zu konzentrieren und zu exportieren, statt an Ort und

11. FIIM/IFPMA. GATT TRIPS and the pharmaceutical industry: a review. Genf, 1995.

12. Challú PM. The consequences of pharmaceutical product patenting. World Competition 1991, Band 15(2), S. 65126.

13. Nogues J. (siehe Anmerkung 4 und 5).

14. Rapp R., Rozek R. Benefits and costs of intellectual property protection in developing countries. Journal of World Trade 1990, Band 24, S. 75-102. 
Stelle zu produzieren oder Lizenzen zu erteilen. ${ }^{15}$ Nach Ansicht anderer wird die Produktion weiterhin in den Entwicklungsländern erfolgen. Es ist heute schwierig, vorherzusehen, was geschehen wird; sehr wahrscheinlich werden die Marktgrösse und das Investitionsklima entscheidende Faktoren für den Technologietransfer sein.

\section{$\square$ Wird das TRIPS-Abkommen die Arzneimittelpreise steigern?}

Eine Reihe lokaler Firmen werden es vorziehen, ihre Anstrengungen auf die Herstellung von Generika (das heisst Medikamente ohne Patent) zu konzentrieren. Diese Tendenz könnte zu einer Senkung der Preise für diese Erzeugnisse führen. Hingegen wird der Preis der patentgeschützten Produkte steigen, weil sie eine Monopolstellung haben werden. ${ }^{16}$ Da die Patente in den meisten Fällen in den Händen der multinationalen Firmen liegen, werden sich die Entwicklungsländer vor Monopolsituationen auf ihrem Binnenmarkt, mit einem Ertragstransfer in die Industrieländer gestellt sehen. Die wenigen erhältlichen Informationen lassen darauf schliessen, dass die Länder, welche die Patente strenger schützen, höhere Erträge transferieren als die Länder mit einem geringen Patentschutzniveau. ${ }^{17}$ Zum anderen werden die bedeutenden Preissenkungen, die sich bei der Einführung von Generika nach Auslaufen eines Patents ergeben, mit der Erhöhung der Patentdauer verzögert werden.

\section{3. ÜBRIGE ENTWICKLUNGSLÄNDER}

Auch bei dieser Ländergruppe werden sich eine Reihe vorhersehbarer Folgen auf die Arzneimittelpreise ergeben. Wie bei den Entwicklungsländern der vorhergehenden Gruppe wird das TRIPS-Abkommen keine sofortige und direkte Auswirkung auf die Politiken der unentbehrlichen Arzneimittel haben, da die meisten dieser Medikamente nicht unter Patentschutz stehen. In den kommenden Jahren wird sich jedoch das Problem der neuen Medikamente stellen. Angesichts der Konsumentwicklung und des Bedarfs an immer höher entwickelten Arzneimitteln könnten die Kosten für die Gesundheitsbudgets und für die Bevölkerung, die den grössten Teil ihres Konsums direkt finanziert, steigen.

\section{EINE BEDEUTENDE HERAUSFORDERUNG FÜR DIE ZUKUNFT}

Das TRIPS-Abkommen stellt ein wichtiges Zugeständnis der Entwicklungsländer - zumindest einiger unter ihnen - als Gegenleistung für durch die Uruguay-Runde insgesamt erhaltene Vorteile dar. Wenn dieses Abkommen nicht mit Einsicht angewandt wird, kann es negative Folgen auf den Arzneimittelzugang in den Entwicklungsländern haben und die wenigen Fortschritte, die da und dort in diesen Ländern festgestellt werden konnten, wieder in Frage stellen. Es ist daher dringlich, dass die Regierungen, die internationalen Organisationen,

15. Verma SK. TRIPS, development and transfer of technology. International Review of Industrial Property and Copyright Law, 1996, Band 27(3).

16. WHO/SEARO. The Uruguay Round and health. SEA/CON/14. New Delhi, WHO Regional Office for South East Asia, 1996.

17. Institut universitaire d'études du développement. Effets de l'Uruguay Round sur les pays en développement: synthèse des études par pays. Band 2, Genf, IUED, 1995, S.28 
die Arzneimittelfirmen und alle anderen Akteure des pharmazeutischen Sektors zusammen neue Strategien festlegen, die einen gerechten Zugang zu den Arzneimitteln und zur Gesundheit gewährleisten und gleichzeitig die vom Abkommen gestellten Bedingungen einhalten. Dies ist eine bedeutende Herausforderung für die kommenden Jahre. Die Hauptausrichtungen der Überlegungen könnten sich um folgende Themen entwickeln :

๖ Wie lässt sich die Umsetzung angemessener Arzneimittelpolitiken in den Entwicklungsländern beschleunigen, wobei es das Hauptziel dieser Politiken ist, den grösstmöglichen Vertrieb qualitativ guter unentbehrlicher Arzneimittel zu möglichst geringen Kosten und ihren rationellen Gebrauch zu ermöglichen? Da die meisten unentbehrlichen Medikamente nicht unter Patentschutz stehen (Generika) und daher zu wettbewerbsfähigen Preisen auf dem internationalen Markt erhältlich sind, dürften die neuen Bestimmungen im TRIPS-Abkommen keine sofortige Auswirkung auf die Preise und die Verfügbarkeit dieser Medikamente haben.

口 Wie lässt sich der Zugang zu den neuen Arzneimitteln (Antiretroviralmittel, Tuberkulosebekämpfungsmittel, usw.) verbessern, die nur unter Patentschutz zu relativ hohen Preisen erhältlich sein werden? Und welche Strategien sollten in Zusammenarbeit mit der auf der Forschung beruhenden Pharmaindustrie entwickelt werden, damit die arme Bevölkerung nicht um die Früchte der Arzneimittelforschung gebracht wird und damit die Forschung sich auch den spezifischen Problemen der Entwicklungsländer zuwendet?

口 Welche Mechanismen sind in den Ländern $\mathrm{zu}$ errichten, die bereits eine nationale Pharmaindustrie haben, um die Bemühung der Industrien und Regierungen auf die Forschung und Entwicklung auszurichten und eine wirkliche Innovationskapazität im pharmazeutischen Bereich zu schaffen?

口 Wie lassen sich die durch das Abkommen und die Übergangsperiode gebotenen Möglichkeiten am besten nutzen, um in die nationalen Gesetzgebungen Bestimmungen einzuführen, die es erlauben, die Interessen der Volksgesundheit zu wahren? Es wird in der Tat im Abkommen anerkannt, dass Massnahmen, die zum Schutz der Volksgesundheit notwendig sind, von den Ländern getroffen werden können, sofern sie mit den Artikeln des Abkommens vereinbar sind.

口 Schliesslich ist es notwendig, damit sich das Abkommen im Sinne des nationalen Gemeinwohls auswirkt und ein Mittel zur Verbesserung der Arzneimittelsituation ist, dass die Länder einen wirksamen Verwaltungsapparat einrichten, der mit den Problemen des geistigen Eigentums beauftragt ist. Ein solcher Apparat wird öffentliche Mehraufwendungen in Staaten, die bereits mittellos sind, nach sich ziehen. Wie kann man ihn gestalten und welches könnte die Rolle der internationalen Gemeinschaft in diesem Bereich sein?

Abschliessend sei gesagt: Obwohl es heute schwierig ist, die Auswirkungen des TRIPS-Abkommens auf die Situation der Arzneimittel in der Welt und den Arzneimittelzugang der Bevölkerung der Entwicklungsländer mit Gewissheit zu messen, ist es klar, dass dieses Abkommen eine neue Herausforderung für die internationale Gemeinschaft darstellt, welche ihre Anstrengungen verdoppeln muss, damit sich die Lage der rund zwei Milliarden Menschen ändert, die keinen Zugang zu den unentbehrlichsten Arzneimitteln haben. 